\title{
Focal neurological deficits from multiple thromboembolic stroke complicating COVID-19
}

\author{
Soo-Hyun Park ${ }^{1}$ and Woo Chang Chun ${ }^{1}$ \\ ${ }^{1}$ Inha University Hospital
}

July 16, 2020

\begin{abstract}
Recent studies reported that the new coronavirus disease (COVID-19) invades the central or peripheral nervous system by various mechanisms including angiotensin converting enzyme-2 receptors, blood-brain barrier injury, and immune injury. The possibility that COVID-19 can occur due to the mechanism of thromboembolic stroke, as a result central nervous system, was confirmed by brain images and laboratory test. What we need to note is that COVID-19 can also appear as a focal neurological defect. In addition, if the COVID-19 patient has a high D-dimer and an inflammatory maker, clinicians must be aware of multiple thromboembolic strokes as well as the occurrence of ischemic stroke caused by large vessel stroke.
\end{abstract}

\section{What's already known about this topic?}

Because the COVID-19 invades the central or peripheral nervous system by various mechanisms including angiotensin converting enzyme-2 receptors, blood-brain barrier injury, and immune injury, the COVID-19 develop neurological symptoms. As a result of the virus penetrating the central nervous system, ischemic stroke can occur.

What does this article add?

Recent studies found that COVID-19 was associated with large vessel stroke. Our study shows that COVID19 can develop focal neurologic deficit due to thromboembolic cause.

Focal neurological deficits from multiple thromboembolic stroke complicating COVID-19

Dear editor,

We are still fighting the new coronavirus disease (COVID-19) worldwide. Some patients with the COVID-19 develop neurological symptoms.1 Recent studies reported that the virus invades the central or peripheral nervous system by various mechanisms including angiotensin converting enzyme- 2 receptors, blood-brain barrier injury, and immune injury.2 Ischemic stroke can occur, as a result of the virus penetrating the central nervous system. The average time of stroke after COVID-19 is about 12 days. 3 The etiology of stroke after COVID-19 diagnosis are diverse. Recent studies found that COVID-19 was associated with large vessel stroke.4 I have experienced a strong association between multiple thromboembolic stroke and COVID-19.5 An 82-year-old woman with known diabetes mellitus and hypertension was admitted to a tertiary hospital with fever $\left(38.0^{\circ} \mathrm{C}\right)$, cough, and diarrhea for two days. One week before hospitalization, her daughter was diagnosed with COVID-19. At admission, her chest X-ray showed multifocal infiltrates in both lungs, and chest computed tomography (CT) showed peripherally distributed patchy ground-glass opacification. Nasopharyngeal swab tested positive for severe acute respiratory syndrome coronavirus 2 on real-time 
reverse transcription-polymerase chain reaction assay. Laboratory findings revealed elevated $\mathrm{C}$ reactive protein $(3.76 \mathrm{mg} / \mathrm{dL}$; normal 0.0-0.5 mg/dl), elevated D-dimer $(16.67 \mathrm{mg} / \mathrm{mL}$; normal 0.0-0.5 $\mu \mathrm{g} / \mathrm{ml})$, normal leukocyte count $\left(7420 / \mathrm{mm}^{3}\right.$; normal $4.0 \times 10^{3}-10.0 \times 10^{3} /$ microliter $)$, and normal prothrombin time (12.7s; normal 11.0-15.0s). Initial treatment involved oral lopinavir/ritonavir $(200 \mathrm{mg} / 500 \mathrm{mg}, 2$ tablets, every day) and hydroxyquinine (200mg, 1 tablet, two times a day) and subcutaneous low molecular weight heparin (40mg, every day). However, fever persisted, and her chest X-ray findings worsened. Intravenous immunoglobulin $(0.3 \mathrm{~g} / \mathrm{kg})$ was added, including oxygen $(2 \mathrm{~L} / \mathrm{min})$ through nasal prongs. On the $8^{\text {th }}$ day of hospitalization (11 days after COVID-19), the patient abruptly complained of weakness and numbness in the right arm. The initial National Institutes of Health Stroke Scale score was 2, with power grade 4+ and decreased sensation in the right arm. The other neurological examination were normal. Brain CT showed a focal low-density lesion in the right cerebellum (Figure 1). The patient's symptoms were mild, and no intravenous alteplase was administered. Brain magnetic resonance image showed multiple high signal intensity lesions with low apparent diffusion coefficient value in the right cerebellum (Figure 2), left precentral gyrus (Figure 3), and left fronto-parietal cortex (Figure 4). Brain magnetic resonance angiography showed no steno-occlusive lesions (Figure 5). The main lesion of the left precentral gyrus caused weakness and numbness of the right arm. The medicine was added to aspirin, clopidogrel, and statin. Trans-thoracic echocardiography and 24h Holter monitoring was normal. The patient's neurological symptoms improved accompanied by rapid diagnosis, medication, and rehabilitation. Elevated CRP and D-dimer may be the main cause of stroke in COVID-19, 6 as they represent an active inflammatory state and abnormalities of the coagulation pathway. 7,8 Our patient elevated CRP and D-dimer, and was no clear abnormalities in other test for stroke mechanism. Focal neurological symptoms occurred after our patient had been managed for COVID-19 for 11 days. If the COVID-19 patient occur neurological symptoms with high D-dimer and an inflammatory maker, clinician be aware of stroke occurrence.

\section{References}

1. Mao L, Jin H, Wang $\mathrm{M}$, et al. Neurologic manifestations of hospitalized patients with coronavirus disease 2019 in Wuhan, China.JAMA Neurol . 2020;77:683-690. https://papers.ssrn.com/sol3/papers.cfm?abstract_id=3550025

2. Wu Y, Xu X, Chen Z, et al. Nervous system involvement after infection with COVID-19 and other coronaviruses. Brain Behav Immun . 2020;87:18-22.

3. Li Y, Wang M, Zhou Y, et al. Acute cerebrovascular disease following COVID-19: a single center, retrospective, observational study.SSRN Electronic Journal. 2020. https://doi.org/10.1001/jamacardio.2020.1286

4. González-Pinto, T., Luna-Rodriguez, A., Moreno-Estebanez, A., et al. Emergency room neurology in times of COVID-19: malignant ischaemic stroke and SARS-CoV-2 infection. Eur J Neurol.https://doi.org/10.1111/ene.14286

5. Goh Y, Beh DL, Makmur A, Somani J, Chan AC. Pearls and Oysters: Facial nerve palsy as a neurological manifestation of Covid-19 infection. Neurology . 2020

6. Divani AA, Andalib S, Di Napoli M, et al. Coronavirus disease 2019 and stroke: clinical manifestations and pathophysiological insights.J Stroke Cerebrovasc . 2020;04941.

7. Madjid M, Safavi-Naeini P, Solomon SD, Vardeny O. Potential Effects of Coronaviruses on the Cardiovascular System: A Review. JAMA Cardiol. 2020. https://doi.org/10.1212/WNL.0000000000009863

8. Klok FA, Kruip MJHA, Van der Meer NJM, et al. Incidence of thrombotic complications in critically ill ICU patients with COVID-19. Thromb Res. 2020;191:145-147.

\section{Figure legends}

Figure 1. Non-contrast enhanced brain computed tomography illustrated a focal low-density lesion in the right cerebellum. 
Figure 2. Diffuse weighted imaging showed diffuse high signal intensity in the right cerebellum.

Figure 3. Diffuse weighted imaging showed diffuse high signal intensity in the left precentral gyrus.

Figure 4. Diffuse weighted imaging showed diffuse high signal intensity in the left fronto-parietal cortex.

Figure 5. Brain magnetic resonance angiography showed luminal irregularity in the basilar artery, and mild segmental stenosis in the distal segment.

\section{Acknowledgements}

This work was supported by the National Research Foundation (NRF) of Korea, funded by a Medical Research Center Grant (NRF-2014R1A5A2009392).

\section{Conflict of Interest}

The authors declare that they have no conflict of interest to disclose.

\section{Authorship}

SH Park: Designed and conceptualized the study, and drafted the manuscript for intellectual content. WC Chun: Acquisition of data, and analyzed of data.

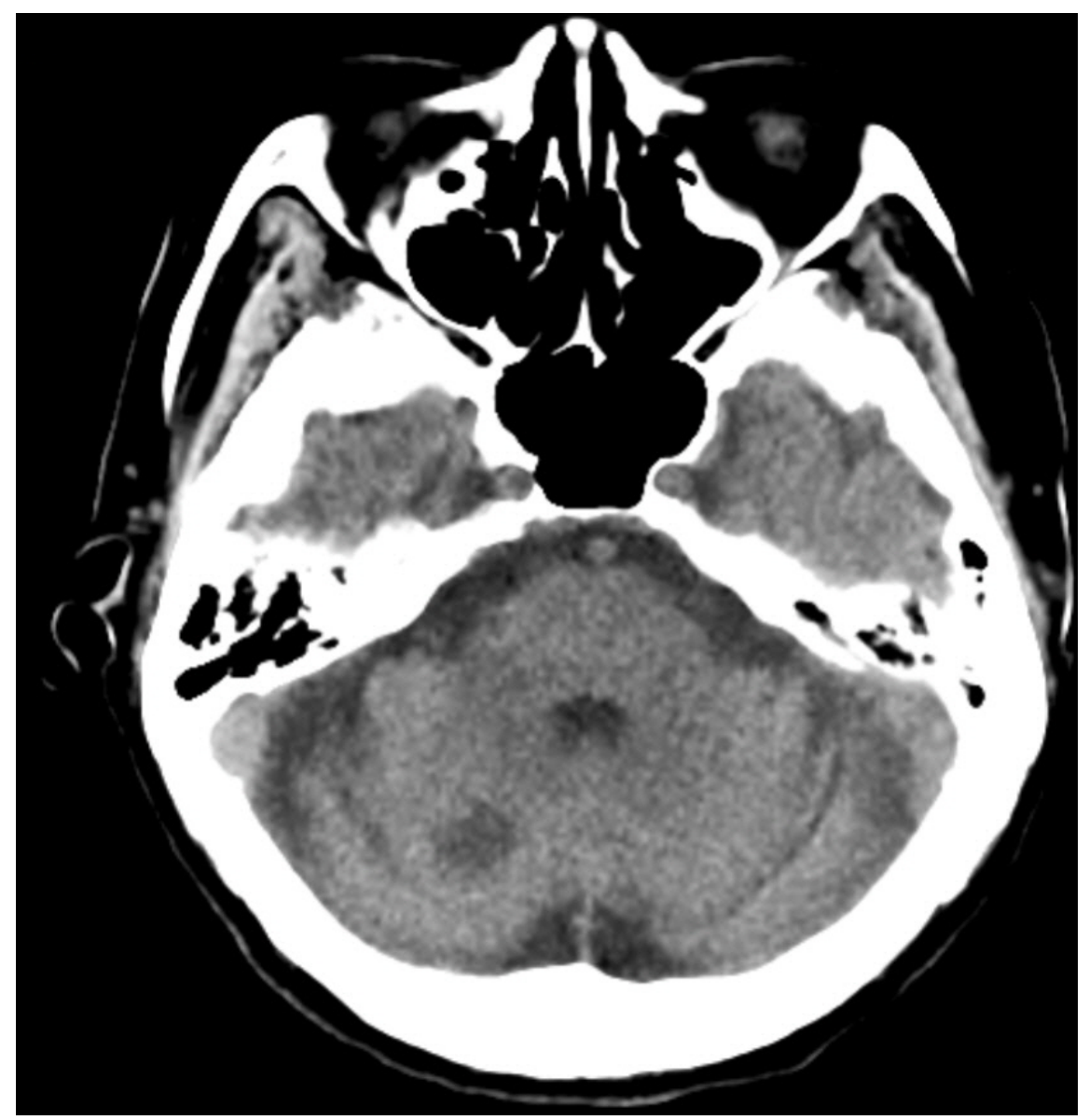




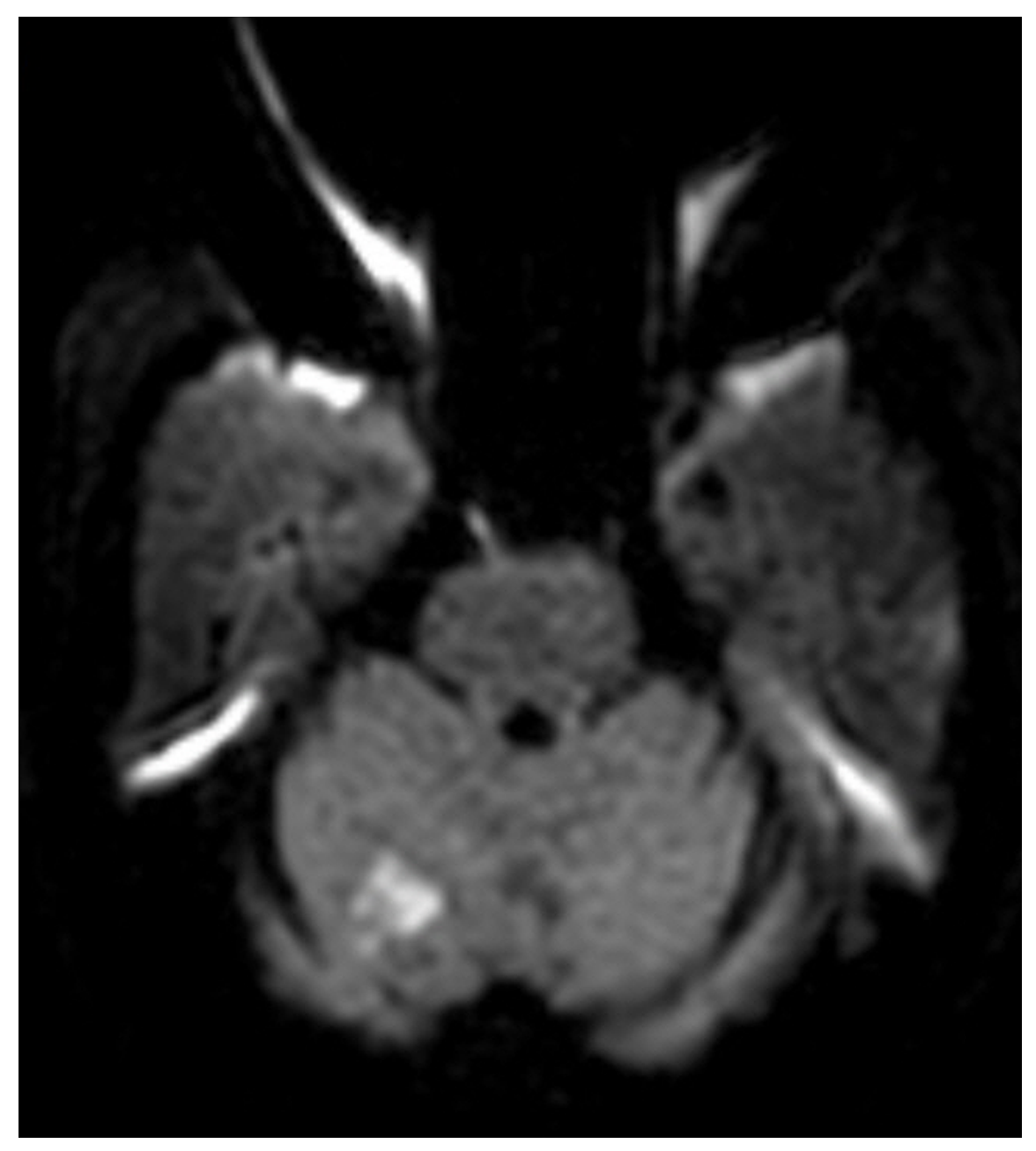




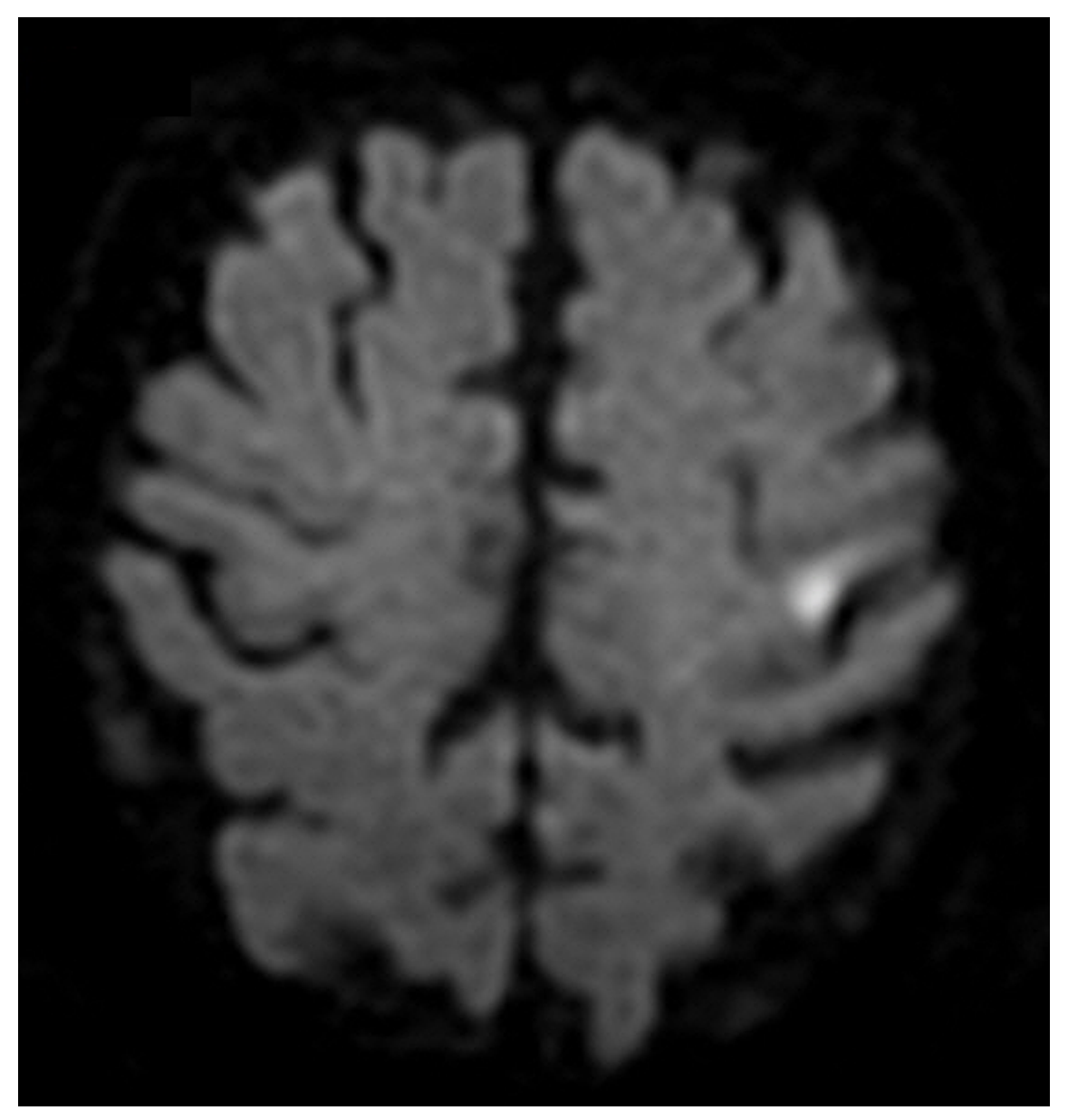



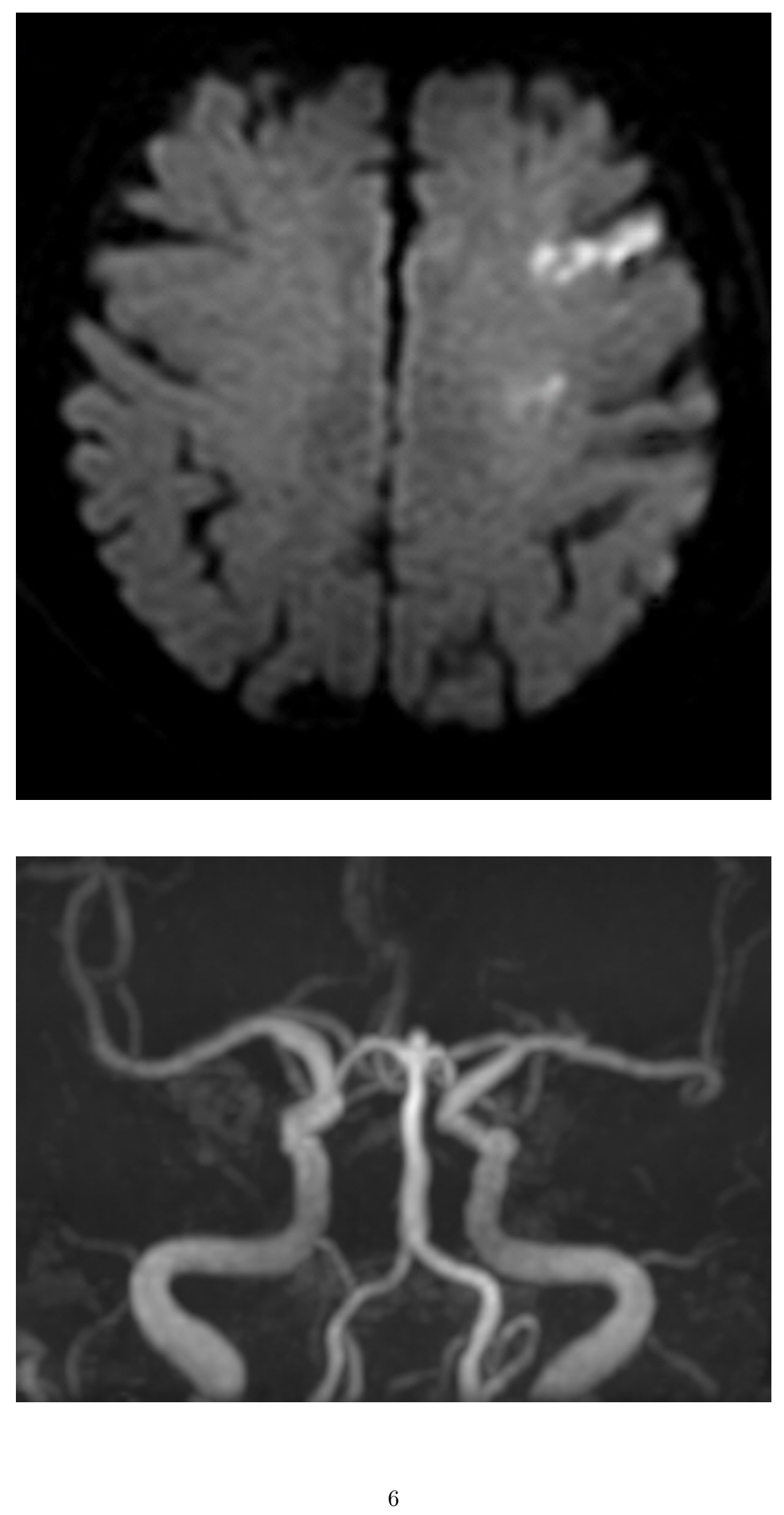